\title{
ADIRF expression reversely correlates with stage progression and involves keratinocyte differentiation in esophageal squamous cell carcinoma
}

\section{Meng Guo}

Air Force Medical University, Fourth Military Medical University

\section{Xiaotong Fan}

Air Force Medical University, Fourth Military Medical University

Shaohua Zhu

Air Force Medical University, Fourth Military Medical University

\section{Xin Zhao}

Air Force Medical University, Fourth Military Medical University

\section{Quan Guo}

Air Force Medical University, Fourth Military Medical University

\section{Lulu Wang}

Air Force Medical University, Fourth Military Medical University

$\mathrm{Na}$ fang

Air Force Medical University, Fourth Military Medical University

\section{Ying Han}

Air Force Medical University, Fourth Military Medical University

Zhiguo Liu ( $\square$ liuzhiguo@fmmu.edu.cn )

Air Force Medical University, Fourth Military Medical University

\section{Research Article}

Keywords: ADIRF, ESCC, tumor progression, Keratinocyte differentiation, SPRR1A

Posted Date: February 3rd, 2022

DOI: https://doi.org/10.21203/rs.3.rs-1291135/v1

License: (c) (i) This work is licensed under a Creative Commons Attribution 4.0 International License. Read Full License 


\section{Abstract}

Background: Aberrant differentiation in esophageal mucosa was crucial for the carcinogenesis and development of esophageal squamous cell carcinoma (ESCC). However, few evidences elucidated the regulatory molecule during ESCC differentiation. We aimed to interpret the correlation between ADIRF expression and the tumor progression and reveal its molecular function in ESCC.

Methods: The ADIRF expression in ESCC was assessed in RNA-seq (RNA sequencing) data from a TCGA (The Cancer Genome Atlas) cohort and immunohistochemically examined in a tissue chip. The correlations with clinical data were assessed in 30 surgical specimens from our center. The regulating effects on cellular function were assessed in the established ADIRF overexpressed and suppressed ESCC cells. The molecular functions of ADIRF were inferenced according to the investigation of the correlated genes.

Results: Both in the mRNA level and in the protein level, expression of ADIRF was decreased in cancerous mucosa than normal tissues, and significantly downregulated in the advanced ESCCs than those in the early stage. ADIRF expression in ESCCs was reversely correlated with the progressing of AJCC stage $(r=-0.41)$ and tumor stage $(r=-0.56)$. The high expression of ADIRF was efficient to distinguish patients with superficial invasion from those with deeper invasion ( $A U C=0.805, P<0.001)$. In vitro, ADIRF showed a suppression role and obviously inhibited the proliferation, migration and division of ESCC cells. According to functional analysis of the coexpressed genes, the ADIRF was putatively associated with the keratinocyte differentiation. Among the keratinocyte differentiation associated genes, SPRR1A, SPRR1B, PKP3, SPRR2D, and FLG were detected to independently correlate with the ESCC prognosis. Thereinto, SPRR1A was with the minimum hazard ratio ( $\mathrm{HR}=0.11,95 \% \mathrm{Cl}: 0.02-0.62)$ and significantly downregulated in the advanced ESCCs.

Conclusions: This study uncovers that ADIRF was reversely correlated with ESCC progression and suppressed malignancy of tumor cells. Moreover, ADIRF was speculated to regulate esophageal keratinocyte differentiation. Hence, ADIRF might be a suppressive factor and with keratinocyte differentiation regulating effect in ESCC.

\section{Background}

Esophageal cancer is one of the most prevalent gastrointestinal carcinomas worldwide, Esophageal squamous cell carcinoma (ESCC) was the common histological types of esophageal cancer and with high incidence rate in China[1, 2]. Absent of an accurate diagnostic method and efficient therapeutic regimen, patients with progressed ESCC carries a dismal prognosis[2, 3]. Endoscopic submucosal dissection and surgery is the predominant choice for the treatment of lesions without deep invasion $[4,5]$. However, without a sufficient knowledge of ESCC characteristics, diagnosis of early esophageal lesions and intervention at early stage was difficultly achieved. The developing and progressing of ESCC is associated with multistage aberrations at genomic and genetic level, which resulted in abnormal 
molecular expression and terminal differentiation program [6]. According to exploration of the differentially expressed genes between each pathological stage and investigating their potential functions, the specifically evidences would facilitate the comprehensive understanding of ESCC.

Abnormal tumor differentiation is the important histopathological feature in squamous cell carcinoma and the differentiation grade is a decisive factor in the AJCC (American joint Committee on Cancer) staging system for ESCC. In multiple squamous cell carcinomas, including but are not limited to ESCC, the differentiation grade has been thought to impact treatment sensitivity and independently affected the overall survival of patients $[7,8]$. Keratinocyte differentiation of esophageal squamous was progressed from basal keratinocytes in the proliferative zone to luminal surface epithelial cells. Tumor development of ESCCs is related to the dysregulation of the squamous cell lineage program and reversed keratinocyte differentiation according to genomic and genetic aberrations. These processes were also be controlled to maintain normal squamous cell development. Differentiation-related genes including SPRR1A, ZNF750, SIM2 and FGFR2 have been reported to regulate ESCC progression[8-11]. However, transcription regulation involved in the keratinocyte differentiation of squamous cell carcinoma remains unclear.

In the study, we focused on adipogenesis regulatory factor (ADIRF), also known as the APM2 or C10orf116, to evaluate its correlation with ESCC progression. Previous studies have indicated that ADIRF was exclusively expressed in adipose tissue and also a variety of other tissues, including subcutaneous, vascular smooth muscle, liver, and kidney tissues[12,13]. Recently, a study revealed that ADIRF potentially regulated chondrogenic differentiation and decreased in human bone marrow mesenchymal stem cells[14]. In prostate carcinoma, ADIRF was report as a biomarker of prostate cancer and the loss was associated with pathological stage progression [15]. In hepatocellular carcinoma, ADIRF was associated with malignant transformation and cisplatin-resistant in both in serum and the tissue $[16,17]$. In squamous cell carcinoma like phenotype of urothelial carcinoma, ADIRF was specifically downregulated and treated as a putative mediator [18]. Those studies suggested a nonnegligible effect of ADIRF in carcinomas and the potential impact in squamous cell carcinoma differentiation, however, the expression characteristics and the correlation with tumor progression in ESCC have not been studied.

Therefore, we will examine the expressional characteristic of ADIRF in the ESCC samples and investigated the correlations between the ADIRF expression and the clinical features. To detect the effects on tumor progression, the implications of ADIRF in ESCC cells growth and motility will be assessed. Finally, we will collect the ADIRF co-expressed genes and excavate their associated core functions in the ESCC. We hope these studies will elucidate the potential roles of ADIRF in the ESCC.

\section{Methods}

\section{Patients and samples}

Between April 2014 and October 2015, 30 patients histologically diagnosed with ESCC and performed esophagectomy in Xijing Digestive Disease Hospital were retrospectively recruited in this study. The clinical information of corresponding patients including were collected. Among which, there were 28 
patients with clear information of differentiation degree and pathological stages. To preliminarily detect the protein expression, a tissue chip contained 40 cases of paired ESCC and precancerous tissues were obtained from technical service company (ES-kx801b, Kexin, Xi'an). The study was approved by the ethics committee of Xijing Hospital and written informed consent was obtained from all patients.

\section{Immunohistochemical Analysis}

The surgically resected tissues contained abundant tumor cells were fixed in paraformaldehyde and embedded in paraffin, then sectioned into $4 \mathrm{~mm}$ slices. Individual tissue slices and tissue chip were incubated with rabbit monoclonal primary anti-ADIRF antibody (HPA026810, Merck, Germany) at $4{ }^{\circ} \mathrm{C}$ overnight, incubated with HRP conjugated Goat anti-mouse rabbit IgG secondary antibody (GK500710, Gene Tech Co., Shanghai) at room temperature for $40 \mathrm{~min}$ and then incubated with DAB Peroxidase (HRP) substrate. Immunohistochemical (IHC) staining for each slice was rendered as IHC score by taking staining extent (positive areas) and intensity into account. The immunostaining intensity was ranked as low staining (1), week staining (2), normal staining (3) or strong staining (4). The staining extent was defined as 1 for $0-25 \%$ positive areas, 1.5 for $26-50 \%$ positive areas, 2 for $51-75 \%$ positive areas, or 2.5 for $76-100 \%$ positive areas. The product of staining extent and intensity was calculated as a IHC score for further analysis.

\section{RNA-Seq data collection}

We obtained RNA-Seq data and the corresponding clinical records of esophageal squamous cell carcinoma patients (ESCC) of TCGA from cBioPortal for Cancer Genomics (http://cbioportal.org)[19]. The data was filtrated base on whether the mRNA expression (RNA Seq V2 RSEM), pathological information and tumor stage were clearly recorded. Collectively, there were 94 samples included for genes expression analysis and 92 samples collected for the stage study, respectively. Among which, there were 83 patients with clear information of differentiation degree and pathological stages.

\section{Bioinformatics}

We assessed the correlation between genes using the R package ggcorrplot. The functional interpretation, the Gene Ontology (GO) functional enrichment analysis and Kyoto Encyclopedia of Genes and Genomes (KEGG) pathway enrichment analysis were performed using the string data-base (string-db.org)[20]. The protein-protein interaction network (PPI) and the most significant hub genes then visualized using Cystoscape software [21].

\section{Cell lines construction}

There were three ESCC cell lines involved in the study. The TE-1 and KYSE150, were cultured in RPMI-1640 medium (cat. no. 11875093, Thermo Fisher Scientific, Inc. United States) and KYSE30 was cultured in DMEM medium (cat. no. 11965092, Thermo Fisher Scientific, Inc. United States) both medium supplemented with 10\% fetal bovine serum (cat. no. 10099141, Thermo Fisher Scientific, Inc. United States). Lentiviral production was using $293 \mathrm{~T}$ cells and followed the Addgene protocol 
(https://www.addgene.org/protocols/lentivirus-production/). The ESCC cells were infected with harvested lentiviral and cumulatively screened in puromycin $(2 \mathrm{ug} / \mathrm{mg})$ for 7 days to obtain stable cell lines.

\section{RT-PCR}

The mRNA levels of ADIRF in the conducted cell lines were validated by real-time polymerase chain reaction (RT-PCR). Total RNA was extracted from the cell pellets using RNA extraction Kit (cat. no. 74204, Qiagen, Germany) and performed reversely transcription to synthesize cDNA according to the (cat. no. K1681, Invitrogen, Thermo Fisher Scientific, Inc. United States). The PCR reaction system contained cDNA and the ADIRF-specific primers were mixed with SYBRß Green regent (cat. no. QPK-212, TOYOBO, Japan). The PCR signals were amplificated and detected in a real-time fluorescence quantitative PCR instrument (Applied Biosystems ${ }^{\mathrm{TM}}$ 7500, Thermo Fisher Scientific, Inc. United States) and normalized by GAPDH. The used primers are listed as follow: shRNA-70-F:

CCGGCATCGACAAGACTGCTAACCACTCGAGTGGTTAGCAGTCTTGTCGATGTTTTTG; shRNA-70-R: AATTCAAAAACATCGACAAGACTGCTAACCACTCGAGTGGTTAGCAGTCTTGTCGATG; shRNA-69-F: CCGGCACCCAGGAAACCATCGACAACTCGAGTTGTCGATGGTTTCCTGGGTGTTTTTG; shRNA-69-R: AATTCAAAAACACCCAGGAAACCATCGACAACTCGAGTTGTCGATGGTTTCCTGGGTG; ADIRF-RT-F: AGACTGCTAACCAGGCCTCT; ADIRF-RT-R: CCGAATTTGGGAGGCTAGGA; GAPDH-RT-F: GGAGCGAGATCCCTCCAAAAT; GAPDH-RT-R: GGCTGTTGTCATACTTCTCATGG.

\section{Cell culture and functional assay}

For the cell viability assay, cells $\left(2 \times 10^{3}\right)$ were seeded in 96 well plates and added cell counting kit-8 regent (cat. no. CK04, Dojindo, Japan) after cultured for 48-96h. Cell viability was assessed after incubation of $2 \mathrm{~h}$ and measured the absorbance at $450 \mathrm{~nm}$. For the colony formation assay, cells $\left(1 \times 10^{3}\right)$ were seeded into each well of a 6 -well and cultured 2 weeks to form colonies. The formatted colonies were fixed with formaldehyde and stained with saturated crystal violet solution for the counting and evaluation. For the transwell migration assay, cells $\left(1 \times 10^{7}\right)$ were placed on the upper layer of a cell culture insert with permeable membrane (cat. no. 353504, BD, United States) and a solution containing the corresponding serum-free medium is placed below the cell permeable membrane. After an incubation of 24-48h, the cells that have migrated through the membrane are stained with saturated crystal violet solution for the assessment. For the scratch healing test, the fused monolayer of cells was uniformly scratched and artificially created a blank area as a " scratch ". The cells at the edge of the scratch gradually migrated into the blank area and allow the scratch to heal. The rate of scratch healing showed as reduction of width and represented the rate of cell migration.

\section{Statistical analysis.}

Survival curves were plotted using a Kaplan-Meier analysis and compared using the log-rank test in GraphPad Prism 8. The mRNA expression between each group were compared by ANOVA analysis. The mRNA expression and the IHC score between the paired samples were assessed using a paired $t$ test. The correlations between clinical characteristics and gene expression were analyzed by regression and shown 
as Pearson correlation. The diagnostic performance was shown as ROC curve and AUC (area under the curve). The ROC curve analysis and AUC calculation were performed in MedCalc (MedCalc Software Ltd.). The survival analyses were performed in Graphpad Prism version 8.0 (GraphPad Software, San Diego, US). Both t test and ANOVA analysis were performed in SPSS 19.0 (IBM, Inc., New York, US). A P value of less than 0.05 was considered statistically significant.

\section{Results}

\section{ADIRF expression was depressed in cancerous tissues and downregulated in the advanced ESCC}

We firstly assessed the mRNA expression of ADIRF in a ESCC RNA-Seq data set and detected that the ADIRF was heterogeneously expressed among the AJCC stages (Figure 1A). Comparing to the paracarcinomas, the expression was significantly reduced in the tumor tissues (Figure 1B). A similar trend of ADIRF downregulation in carcinomas was also detected between the paired tissues (Figure 1C). Assessment of the clinical features in 83 well-documented patients suggested that the ADIRF expression was positively correlated with patients' age and negatively correlated with the grade and AJCC stage (Figure 1D). To evaluate the relevance to progress, we additionally investigated the mRNA expressions between the AJCC stages and found that ADIRF was obviously downregulated in the stage II and stage III/IV $(P<0.01$ and $P<0.001)$ comparing to that in the early stage (Stage I) (Figure 1E). Additional regression analysis in all patients revealed that the transcriptional level of ADIRF was significantly and negatively correlated with pathological stage progressing $(r=-0.25, P=0.016)$ (Figure 1F) and differentiation degree $(r=-0.24, P=0.028)$ (Figure 1G). Similar as the trend between the AJCC stages, the expression of ADIRF was significantly downregulated in the T2 patients than those with T1 disease (Figure 1H). Due to the limited sample size, we didn't detect significant prognostic associations of ADIRF expression. No differences in overall survivals and progression-free survivals (PFS) were detected between the low and high expression groups $(P>0.05$, Figure $1 \mathrm{I}$ and $1 \mathrm{~J})$. But still there was a trend towards prolonged PFS in patients with high expression of ADIRF (28.11 vs 15.91 months, HR=0.72).

According to the IHC analysis in a tissue chip, we compared the protein expression of ADIRF between the ESCCs and normal mucosa. Consistent with the difference in mRNA level, the ADIRF protein was tends to positively express in the para-carcinomas tissues rather than that in the carcinomas (Figure 1K). Between the paired tissues, the expressional levels of ADIRF were dramatically downregulated in the ESCCs comparing to those in the normal para-cancerous tissues $(P<0.0001$, Figure $1 \mathrm{~J})$.

\section{ADIRF expression was reversely correlated with tumor progression and abled to indicate the early stage in ESCC}

To verify the expressional characteristics of ADIRF in ESCCs, we performed immunostaining of ADIRF in 30 surgically resected specimens. Among the tissues with different invasion depths, the protein expression of ADIRF was obviously heterogeneous and tends to be suppressed in the cases with deeper invasion (Figure 2A). Assessing the correlations between the ADIRF immunostaining and the clinical characteristics showed that significant differences were shown in the invasion depth $(P<0.01)$, AJCC 
stage $(P<0.01)$ and tumor stage $(P<0.01)$ between the patients with low and high expression of ADIRF. Meanwhile, there were no differences in the terms of age, gender, tumor location, differentiation degree and lymph node stage (Table 1). Regression analysis revealed that ADIRF expression were significantly and reversely correlated with AJCC stage $(P<0.05)$ and tumor stage $(P<0.001)$, which results were consistent with the findings in the RNA-Seq data (Figure 2B and Supplementary table 1). Further analysis of the relevance in all specimens indicated that ADIRF expression were strongly correlated with the progression of AJCC stage $(r=-0.41)$ and tumor stage ( $r=-0.56)$ (Figure $2 \mathrm{C}$ and $2 \mathrm{D}$ ).

We further compared the protein levels of ADIRF between each tumor stage and found that the protein expression of ADIRF was significantly downregulated in stage II $(P<0.05)$ and stage III $(P<0.01)$ than that in stage I (Figure 2E). Moreover, comparison of ADIRF staining between early and progressed disease revealed that the protein expression of ADIRF was obviously suppressed in the advanced stages (stage $\mathrm{II} / \mathrm{III})$ than that in the early stage (stage I) $(\mathrm{P}<0.01)$ (Figure $2 \mathrm{~F}$ ). Therefore, we conducted a predication model using ADIRF expression to indicate advanced diseases. When the associated criterion of IHC score was more than $\quad 4$, the ADIRF immunostaining in ESCC tissues showed excellent performance to distinguish early stages from advanced stages (AUC $=0.805,95 \% \mathrm{Cl}: 0.620-0.926$ ) with a Youden index $\mathrm{J}$ of 0.55 , sensitivity of 90.00 , and specificity of 65.00 (Figure $2 G$ ).

\section{ADIRF suppressed tumor growth and migration in ESCC cells}

To assess the effect of ADIRF expression on ESCC cells, we established the ADIRF-overexpressed and ADIRF-suppressed (shRNA binding at two different sites) ESCC cell lines in multiple ESCC cells (Figure $3 \mathrm{~A})$. Comparing to the controls, the proliferations were decreased in the ADIRF-overexpressed cell lines (TE-1 and KYSE30). Meanwhile, the proliferations of the cells were significantly increased after ADIRF suppressed (Figure 3B). In the invasiveness analysis, capabilities of invasion were reduced in the three ADIRF-overexpressed cell lines (TE-1, KYSE30, and KYSE150) comparing to that in the controls (Figure 3C). Oppositely, the invasive capabilities were observably enhanced in the correspondingly ADIRF suppressed cell lines (Figure 3D). According to the clonogenic assay, the division abilities of KYSE30 and KYSE150 were restrained after ADIRF overexpressed (Figure 3E). Meanwhile, the division abilities were observably enhanced in the ADIRF-suppressed cells than that in the controls (Figure 3F). We next conducted the scratch healing assay and detected that the migration in the ADIRF-overexpressed cell line (KYSE150) was suppressed comparing to that in the controls (Figure 3G). Meanwhile, the corresponding multisite-ADIRF-inhibited cell line was with activated migration of the than that in the controls (Figure $3 H)$. Those results suggested an inhibiting effect of ADIRF in ESCC cells.

\section{ADIRF was associated with keratinocyte differentiation in ESCC}

According the RNA-seq data from the TCGA cohort, we detected that of 471 genes were significantly correlated with ADIRF in mRNA expression ( $r>0.2$ or $<-0.2, P<0.05)$. Assessing the functional networks of the proteins coded by those ADIRF-correlated genes reveled that most genes were enriched and associated with keratinocyte differentiation (Figure 4A). The gene ontology (GO) analysis showed that the most enriched terms in cellular component were cornified envelope, desmosome and keratin filament 
(Figure 4B); the most enriched terms in biological process were desmosome organization, establishment of skin barrier and peptide cross-linking (Figure 4C); the three enriched terms in molecular functions were all correlated with cadherin binding in cell-cell adhesion (Figure 4D). The tissue specificity analysis revealed that the most of those genes were expressed in oral mucosa, keratinocyte and tongue (Figure $4 \mathrm{E})$.

We further divided the ADIRF-correlated genes into positive correlation set (317 genes) and negative correlation set (154 genes). Among the positively correlated genes, the most of those genes were still functionally enriched in keratinocyte differentiation (Figure 4F). The GO analysis indicated that those genes positively correlated with ADIRF were also involved in cornified envelope, desmosome organization and cell-cell adhesion (Figure 4G-4I). According to the pathway analysis, the keratinization was shown as the most significant term (Figure $4 \mathrm{~J}$ ). In the negative correlation set, most genes were specifically expressed in female reproductive system (Figure $4 \mathrm{~L}$ ), and correlated with the key words (KW analysis) of basement membrane, glycosyltransferase, repeat, phosphoprotein, and alternative splicing (Figure 4M).

\section{SPRR1A was correlated with ADIRF and reversely correlated with ESCC progression}

Given the close correlation between ADIRF and keratinocyte differentiation and the significant association with ESCC stage of ADIRF, we next assessed the expressional characteristics keratinocyte differentiation associated genes according to the RNA-seq data from ESCC samples (Supplementary table 2). Of 46 genes involved in keratinocyte differentiation were unevenly expressed among AJCC stages (Figure 5A). Interestingly, all those genes were significantly downregulated in advanced stages (III/IV) compared to that in the earlier stages (I/II) (Figure 5B). Except STK4 (Serine/threonine-protein kinase 4) negatively correlated, other keratinocyte associated genes were positively correlated with ADIRF. Among which, CNFN (Cornifelin) and SPRR1B (Small proline-rich protein IB) showed the strongest correlations ( $r>0.6)$ (Figure $5 \mathrm{C}$ ). Interaction network indicated that SPRR1A (Small proline-rich protein IA) and SPRR1B were located at the regulatory center of keratinocyte differentiation (Figure 5D). Evaluating the correlations between and the AJCC stage and the individual gene expression indicated that most of the keratinocyte differentiation associated genes were reversely correlated with stage progression (Figure $5 E$ ). Statistic indicated there were 12 genes showed significant correlations (absolute $r \geq 0.2$ ) with AJCC stage. Among which, SPRR1A was with the strongest correlation $(r=-0.31, P<0.01)$. To investigated the prognostic associations, we conducted a COX regression analysis including the 12 genes. It revealed that five genes of SPRR1A, SPRR1B, PKP3 (Plakophilin-3), SPRR2D (Small proline-rich protein 2D), and FLG (Filaggrin) were significantly associated with the prognosis in ESCC. Thereinto, SPRR1A was shown as the most significant protective factor for OS ( $\mathrm{HR}=0.115,95 \% \mathrm{Cl}=0.021-0.623)$ (Supplementary table 3). Further investigation indicated that SPRR1A was observably correlated with ADIRF $(r=0.39, P<0.0001)$ (Figure 5F). Comparing to the expression in the stage I, SPRR1A was significantly downregulated in the stages II $(P<0.0001)$ and stage III/ $\mathrm{V}(P<0.001)($ Figure $5 \mathrm{H})$.

\section{Discussion}


Multiple previous studies demonstrated the genomic and epigenomic characteristics of ESCCs, inadequately, the early events to promote cancerous progression is less known [6]. In the program accompanies neoplasia, the abnormal activation and repression of differentiation-associated genes introduced the prosoplasia process and derived squamous cell differentiation. Identification of early genetic variation in esophageal squamous cell differentiation is critical for identifying the diagnostic marker for ESCCs at early stages, and indicating potential pathogenesis that drive the disease[22]. In the current study, we uncovered that ADIRF was downregulated in the advanced ESCC and reversely correlated with the stage progression. The ADIRF immunostaining in the ESCC tissues enabled to distinguish the patients with early stage from those with the advanced stages. According to in vitro assay, we detected a suppressive role of ADIRF in the growth and invasion of ESCC cells. Moreover, we demonstrated that the ADIRF-correlated genes were primarily involved in keratinocyte differentiation. Hence, we concluded that ADIRF was putatively regulated keratinocyte differentiation in ESCCs.

Diagnosis of early lesions in esophageal mucosa is crucial for optimizing the subsequent treatment. Once neoplasia programmed into advanced disease, the risk of lymphatic metastasis will significantly increase and the cases would be beyond indications of endoscopic treatment[23]. Hence, prospectively evaluation of the degree of tumor progression stage facilitated the clinical management of ESCC. According to the resected specimens, we found that ADIRF expression was significantly and reversely correlated with ESCC stage progression. Moreover, the elevated expression of ADIRF was efficient to indicate the cases in early stage (Figure 2). There were $90 \%$ lesions progressed from early stage to advanced stage once the IHC score of ADIRF less than 4, which should be taken more radical resection or additional adjuvant chemoradiotherapy. On the other side, the in vitro examination demonstrated the suppressor role of ADIRF in ESCC cells. Both in the overexpressed and suppressed cell lines, the effects of ADIRF in functional phenotypes were congruence, which was particularly detected in the migration assay (Figure 3). Those results were consistent with previous studies of ADIRF in urothelial carcinoma and prostate carcinoma $[18,24]$. The study in prostate carcinoma revealed that copy number loss of ADIRF was associated with higher pathological stage, higher clinical stage, and lymph node metastasis, which might partly interpretate the downregulation of ADIRF in the advanced ESCCs.

Aberrant keratinocyte differentiation is an important process in the initiation of squamous carcinoma. Because activities regulating differentiation exhibit altered or reduced capacity in esophageal cancer cells, it is vital to pinpoint those genes that control epidermal proliferation and terminal differentiation to better understand esophageal carcinogenesis. For the first time, we found that ADIRF expression was dramatically correlated with keratinocyte differentiation associated genes in ESCC, which indicating a putative role of ADIRF in regulating keratinocyte differentiation. Among those genes, SPRR1A was the most ADIRF-correlated candidate and the hub of interaction network of keratinocyte differentiation. SPRR1A, also kwon as Cornifin-A, is a keratinocyte protein that enabled to formed an insoluble envelope beneath the plasma membrane. SPRR1A is strictly linked to keratinocyte terminal differentiation $[25,26]$ and strikingly upregulated in the normal esophagus[27]. According our study, SPRR1A was shown as an independent prognostic factor in ESCC and, similar as ADIRF, reversely correlated with stage progression (Figure 5). For the assessment of differentiation-associated genes, an early study using cDNA microarray 
showed that the SPRR1A, SPRR2A and KRT4 were downregulated in ESCC, which consequently supported the expressional characteristic of SPRR1A in our RNA-Seq results[9]. Another prior study focusing on the role of S100A14 in terminal differentiation indicated that the expression pattern of S100A14 is concordant with SPRR1A during calcium-induced differentiation of esophageal cancer cells [28]. Interestingly, our data also showed dramatically correlations between ADIRF and S100A14 $(r=0.59$, $P<0.0001)$ and between SPRR1A and S100A14 $(r=0.734, P<0.0001)$. This high consistency emphasized the association of ADIRF and differentiation moderating.

\section{Conclusions}

In conclusion, we have characterized the reverse correlation between the ADIRF expression and ESCC progression and revealed the putative regulation role of ADIRF in keratinocyte differentiation. What calls for special attention in further research is that the diagnostic role of ADIRF should be verified in a larger sample size and the molecular mechanisms to modulate keratinocyte differentiation need to be elucidated.

\section{Abbreviations}

ADIRF: adipogenesis regulatory factor; AJCC: American joint Committee on cancer; AUC: area under the curve; CNFN: Cornifelin; ESCC: esophageal squamous cell carcinoma; FLG: Filaggrin; GO: Gene Ontology; IHC: Immunohistochemical; KEGG: Kyoto Encyclopedia of Genes and Genomes; KW: key words; PKP3: Plakophilin-3; RNA-seq (RNA sequencing); SPRR1A: Small proline-rich protein IA; SPRR1B: Small prolinerich protein IB; SPRR2D: Small proline-rich protein 2D; STK4: Serine/threonine-protein kinase 4; TCGA: The Cancer Genome Atlas.

\section{Declarations}

\section{Ethics approval and consent to participate}

The study was approved by the ethics committee of Xijing Hospital and written informed consent was obtained from all patients.

\section{Availability of data and materials}

The datasets used and assessed during the current study are available from the corresponding author on reasonable request.

\section{Conflicts of Interest}

All the authors have no conflicts of interest to declare.

\section{Grant support}


This work was supported by National Natural Science Foundation of China $(81572820,81702355)$ and Key Research and Development Program of Shaanxi Province (2018ZDXM-SF-050).

Authors' contributions

$\mathrm{ZL}$ and $\mathrm{YH}$ designed the study. MG assessed the RNA-seq data and performed the bioinformatics analysis. XF and XZ performed the immunohistological examination. SZ and NF collected and interpreted the clinical data. MG, QG and LW cultured the cells and evaluated the cell functions. MG drafted the manuscript. ZL was a major contributor in writing and reviewing the manuscript. All authors read and approved the final manuscript.

Acknowledgements

No.

\section{References}

1. Li J, Xu J, Zheng Y, Gao Y, He S, Li H, Zou K, Li N, Tian J, Chen W, He J: Esophageal cancer: Epidemiology, risk factors and screening. Chin J Cancer Res 2021, 33:535-547.

2. Sung H, Ferlay J, Siegel RL, Laversanne M, Soerjomataram I, Jemal A, Bray F: Global Cancer Statistics 2020: GLOBOCAN Estimates of Incidence and Mortality Worldwide for 36 Cancers in 185 Countries. CA Cancer J Clin 2021, 71:209-249.

3. Merkow RP, Bilimoria KY, Keswani RN, Chung J, Sherman KL, Knab LM, Posner MC, Bentrem DJ: Treatment trends, risk of lymph node metastasis, and outcomes for localized esophageal cancer. $J$ Natl Cancer Inst 2014, 106.

4. di Pietro M, Canto MI, Fitzgerald RC: Endoscopic Management of Early Adenocarcinoma and Squamous Cell Carcinoma of the Esophagus: Screening, Diagnosis, and Therapy. Gastroenterology 2018, 154:421-436.

5. Yamauchi K, Iwamuro M, Nakagawa M, Takenaka R, Matsueda K, Inaba T, Yoshioka M, Toyokawa T, Tanaka S, Kanzaki H, et al: Long-term outcomes of endoscopic versus surgical resection for MMSM1 esophageal squamous cell carcinoma using propensity score analysis. Esophagus 2021, 18:7280.

6. Lin DC, Wang MR, Koeffler HP: Genomic and Epigenomic Aberrations in Esophageal Squamous Cell Carcinoma and Implications for Patients. Gastroenterology 2018, 154:374-389.

7. Hou X, Gu YK, Liu XW, Fu JH, Wang X, Zhang LJ, Luo RZ, Lin P, Yang HX: The impact of tumor cell differentiation on survival of patients with resectable esophageal squamous cell carcinomas. Ann Surg Oncol 2015, 22:1008-1014.

8. Tamaoki M, Komatsuzaki R, Komatsu M, Minashi K, Aoyagi K, Nishimura T, Chiwaki F, Hiroki T, Daiko $\mathrm{H}$, Morishita $\mathrm{K}$, et al: Multiple roles of single-minded 2 in esophageal squamous cell carcinoma and its clinical implications. Cancer Sci 2018, 109:1121-1134. 
9. Luo A, Kong J, Hu G, Liew CC, Xiong M, Wang X, Ji J, Wang T, Zhi H, Wu M, Liu Z: Discovery of Ca2+relevant and differentiation-associated genes downregulated in esophageal squamous cell carcinoma using cDNA microarray. Oncogene 2004, 23:1291-1299.

10. Ana Choi SS, Ko JM, Yu VZ, Ning L, Lung ML: Differentiation-related zinc finger protein 750 suppresses cell growth in esophageal squamous cell carcinoma. Oncol Lett 2021, 22:513.

11. Maehara O, Suda G, Natsuizaka M, Shigesawa T, Kanbe G, Kimura M, Sugiyama M, Mizokami M, Nakai M, Sho T, et al: FGFR2 maintains cancer cell differentiation via AKT signaling in esophageal squamous cell carcinoma. Cancer Biol Ther 2021, 22:372-380.

12. Wang S, Liu J, Zhao W, Wang G, Gao S: Selection of candidate genes for differences in fat metabolism between cattle subcutaneous and perirenal adipose tissue based on RNA-seq. Anim Biotechnol 2021:1-12.

13. Lee SJ, Blanchett-Anderson S, Keep SG, Gasche MB, Wang MM: Tripartite factors leading to molecular divergence between human and murine smooth muscle. PLoS One 2020, 15:e0227672.

14. Jiang Y, Zhang C, Long L, Ge L, Guo J, Fan Z, Yu G: A Comprehensive Analysis of SE-IncRNA/mRNA Differential Expression Profiles During Chondrogenic Differentiation of Human Bone Marrow Mesenchymal Stem Cells. Front Cell Dev Biol 2021, 9:721205.

15. Zhou K, Arslanturk S, Craig DB, Heath E, Draghici S: Discovery of primary prostate cancer biomarkers using cross cancer learning. Sci Rep 2021, 11:10433.

16. Kamimura K, Suda T, Fukuhara Y, Okuda S, Watanabe Y, Yokoo T, Osaki A, Waguri N, Ishikawa T, Sato $\mathrm{T}$, et al: Adipose most abundant 2 protein is a predictive marker for cisplatin sensitivity in cancers. $\mathrm{Sci}$ Rep 2021, 11:6255.

17. Chen HQ, Zhao J, Li Y, He LX, Huang YJ, Shu WQ, Cao J, Liu WB, Liu JY: Gene expression network regulated by DNA methylation and microRNA during microcystin-leucine arginine induced malignant transformation in human hepatocyte L02 cells. Toxicol Lett 2018, 289:42-53.

18. Eriksson P, Aine M, Veerla S, Liedberg F, Sjödahl G, Höglund M: Molecular subtypes of urothelial carcinoma are defined by specific gene regulatory systems. BMC Med Genomics 2015, 8:25.

19. Gao J, Aksoy BA, Dogrusoz U, Dresdner G, Gross B, Sumer SO, Sun Y, Jacobsen A, Sinha R, Larsson E, et al: Integrative analysis of complex cancer genomics and clinical profiles using the cBioPortal. Sci Signal 2013, 6:pl1.

20. Szklarczyk D, Gable AL, Nastou KC, Lyon D, Kirsch R, Pyysalo S, Doncheva NT, Legeay M, Fang T, Bork P, et al: The STRING database in 2021: customizable protein-protein networks, and functional characterization of user-uploaded gene/measurement sets. Nucleic Acids Res 2021, 49:D605-d612.

21. Otasek D, Morris JH, Bouças J, Pico AR, Demchak B: Cytoscape Automation: empowering workflowbased network analysis. Genome Biol 2019, 20:185.

22. Ma S, Bao JYJ, Kwan PS, Chan YP, Tong CM, Fu L, Zhang N, Tong AHY, Qin YR, Tsao SW, et al: Identification of PTK6, via RNA sequencing analysis, as a suppressor of esophageal squamous cell carcinoma. Gastroenterology 2012, 143:675-686.e612. 
23. Oyama T, Inoue H, Arima M, Momma K, Omori T, Ishihara R, Hirasawa D, Takeuchi M, Tomori A, Goda $\mathrm{K}$ : Prediction of the invasion depth of superficial squamous cell carcinoma based on microvessel morphology: magnifying endoscopic classification of the Japan Esophageal Society. Esophagus 2017, 14:105-112.

24. Meng J, Wang LH, Zou CL, Dai SM, Zhang J, Lu Y: C10orf116 Gene Copy Number Loss in Prostate Cancer: Clinicopathological Correlations and Prognostic Significance. Med Sci Monit 2017, 23:51765183.

25. Kartasova T, van Muijen GN, van Pelt-Heerschap H, van de Putte P: Novel protein in human epidermal keratinocytes: regulation of expression during differentiation. Mol Cell Biol 1988, 8:2204-2210.

26. Sark MW, Fischer DF, de Meijer E, van de Putte P, Backendorf C: AP-1 and ets transcription factors regulate the expression of the human SPRR1A keratinocyte terminal differentiation marker. $\mathrm{J}$ Biol Chem 1998, 273:24683-24692.

27. Fagerberg L, Hallström BM, Oksvold P, Kampf C, Djureinovic D, Odeberg J, Habuka M, Tahmasebpoor $\mathrm{S}$, Danielsson A, Edlund $\mathrm{K}$, et al: Analysis of the human tissue-specific expression by genome-wide integration of transcriptomics and antibody-based proteomics. Mol Cell Proteomics 2014, 13:397406.

28. Chen H, Ma J, Sunkel B, Luo A, Ding F, Li Y, He H, Zhang S, Xu C, Jin Q, et al: S100A14: novel modulator of terminal differentiation in esophageal cancer. Mol Cancer Res 2013, 11:1542-1553.

\section{Tables}

Table 1: Clinical characteristics of patients with different ADIRF expression. 


\begin{tabular}{|c|c|c|c|c|}
\hline & & ADIRF-Low & ADIRF-High & $P$ value \\
\hline & & $(n=14)$ & $(n=16)$ & \\
\hline Age & & $60.86 \_10.00$ & 59.69_10.42 & 0.757 \\
\hline Gender & Male & 10 & 10 & 0.605 \\
\hline & Female & 4 & 6 & \\
\hline Location & Middle & 7 & 10 & 0.775 \\
\hline & Middle-lower & 2 & 2 & \\
\hline & Lower & 5 & 4 & \\
\hline Invasion depth & Muscularis mucosa & 1 & 9 & $0.001^{* *}$ \\
\hline & Muscularis propria & 4 & 6 & \\
\hline & Adventitia & 9 & 1 & \\
\hline Differentiation degree & Highly & 3 & 4 & 0.818 \\
\hline & Moderately & 11 & 12 & \\
\hline AJCC stage & 1 & 1 & 10 & $0.007^{* k}$ \\
\hline & II & 7 & 4 & \\
\hline & III & 4 & 0 & \\
\hline & IV & 2 & 2 & \\
\hline Tumor stage & 1 & 1 & 9 & $0.001^{* *}$ \\
\hline & 2 & 4 & 6 & \\
\hline & 3 & 9 & 1 & \\
\hline Lymph node stage & $x$ & 1 & 1 & 0.128 \\
\hline & 0 & 7 & 13 & \\
\hline & 1 & 2 & 0 & \\
\hline & 2 & 2 & 0 & \\
\hline & 3 & 2 & 2 & \\
\hline
\end{tabular}

**: $P<0.01$.

Figures 
Figure 1
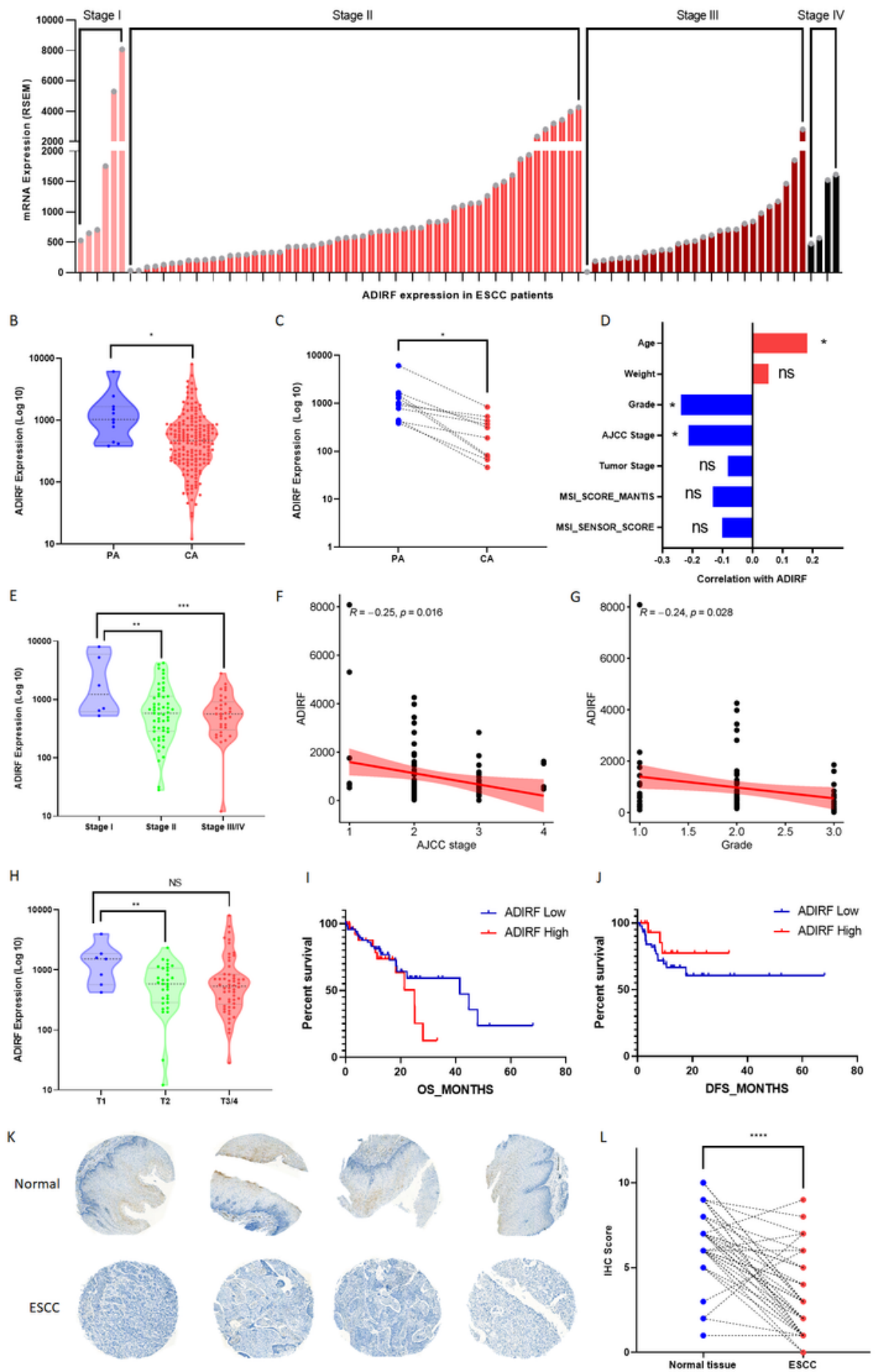

Figure 1

ADIRF expression was downregulated in the ESCCs and reversely correlated with progression on ESCC stage. The mRNA expression of ADIRF mRNA was heterogeneously expressed among various ESCC stages (A), meanwhile, significantly reduced in the ESCCs comparing to the normal esophageal mucosa (B) and paired paracancerous tissues (C). ADIRF was significantly correlated with age, grade and AJCC stage in $\operatorname{ESCC}(D, F, G)$. The mRNA expression of ADIRF was downregulated as tumor progressing from 
early to advanced stages $(E)$ with increased local invasion depth $(H)$. There was a trend towards prolonged PFS in patients with higher expression of ADIRF (I), but no significant difference in overall survivals between the different ADIRF expressions (J). According to immunostaining in tissue chip, the protein level of ADIRF was also dramatically downregulated in the matched ESCCs comparing to the paired normal tissues (K and L). $\left({ }^{*}: \mathrm{P}<0.05 ;{ }^{* *}: \mathrm{P}<0.01 ;{ }^{* * *}: \mathrm{P}<0.001\right)$.

\section{Figure 2}

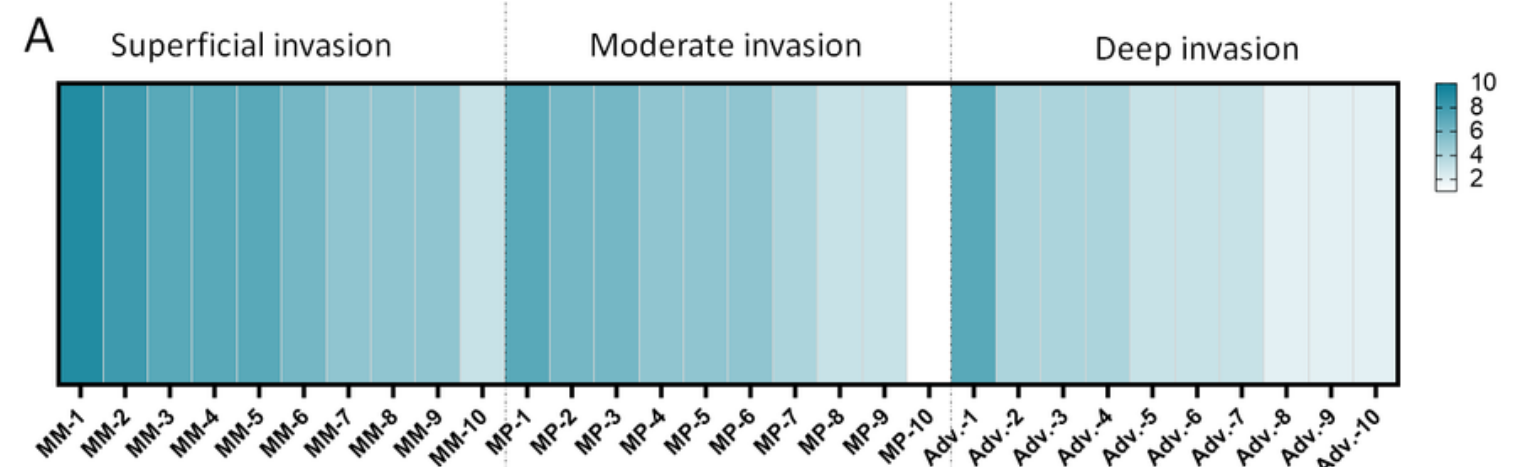

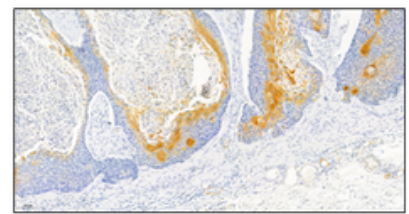
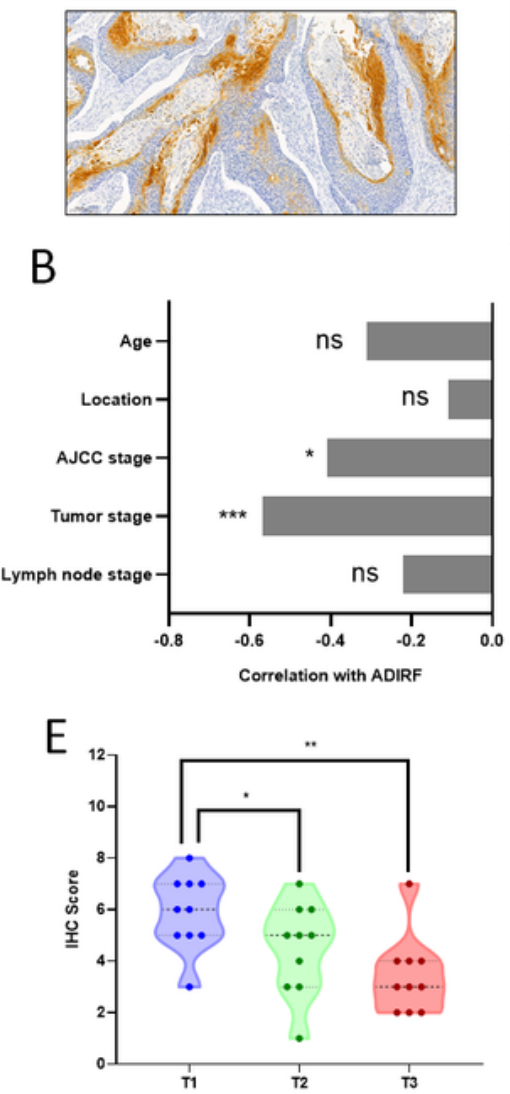
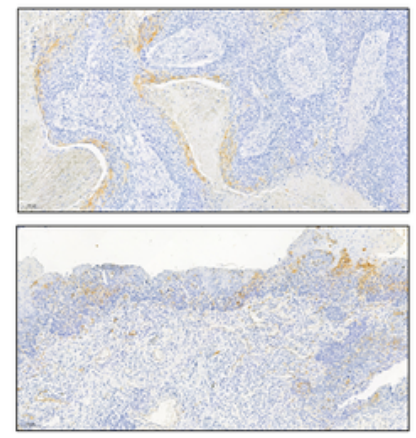

C
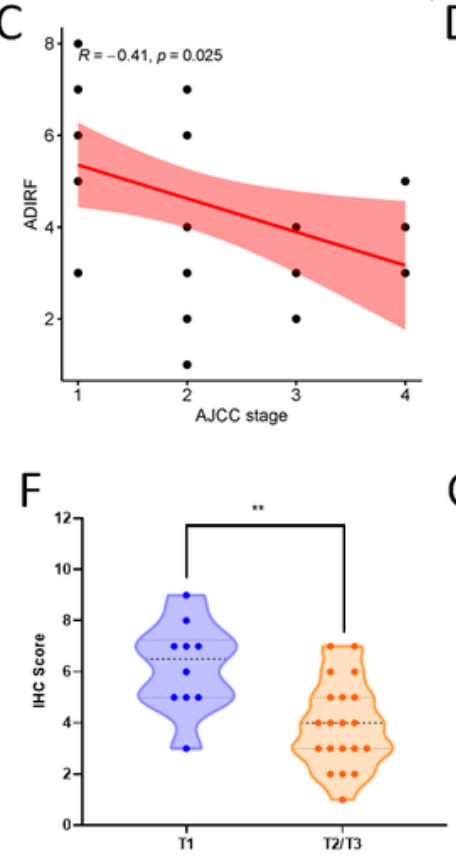
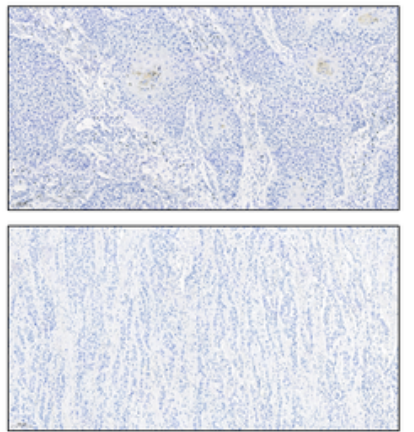

D
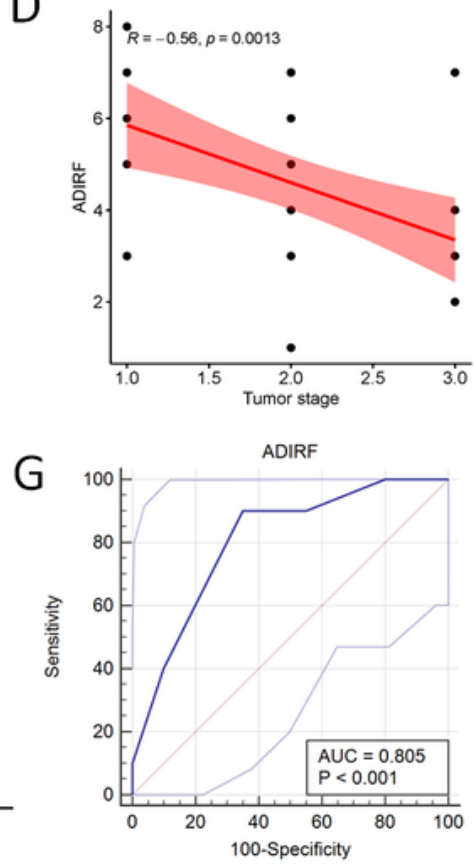

Figure 2 


\section{Downregulation of ADIRF was reversely correlated with tumor progression and abled to distinguish the}

early ESCC. The expressions of ADIRF were obviously heterogeneous among ESCCs with different invasion depths, the representative immunostaining images were shown $(A)$. The protein expression of ADIRF reversely correlated with the stage progression, as well as the tumor stage progression (B-D). The protein expressions of ADIRF were significantly downregulated in carcinomas with moderate invasion (T2) and deep invasion (T3) compared to those with superficial invasion (T1) (E). In addition, the protein expressions of ADIRF in the superficially invasive diseases (T1) were significantly higher than that in the deeper invaded diseases (T2/T3) (F). The immunostaining degree was efficient to distinguish the early stages from the advanced stages $(G) .\left({ }^{*}: P<0.05 ;{ }^{* *}: P<0.01 ;{ }^{* * *}: P<0.001\right)$ 


\section{Figure 3}

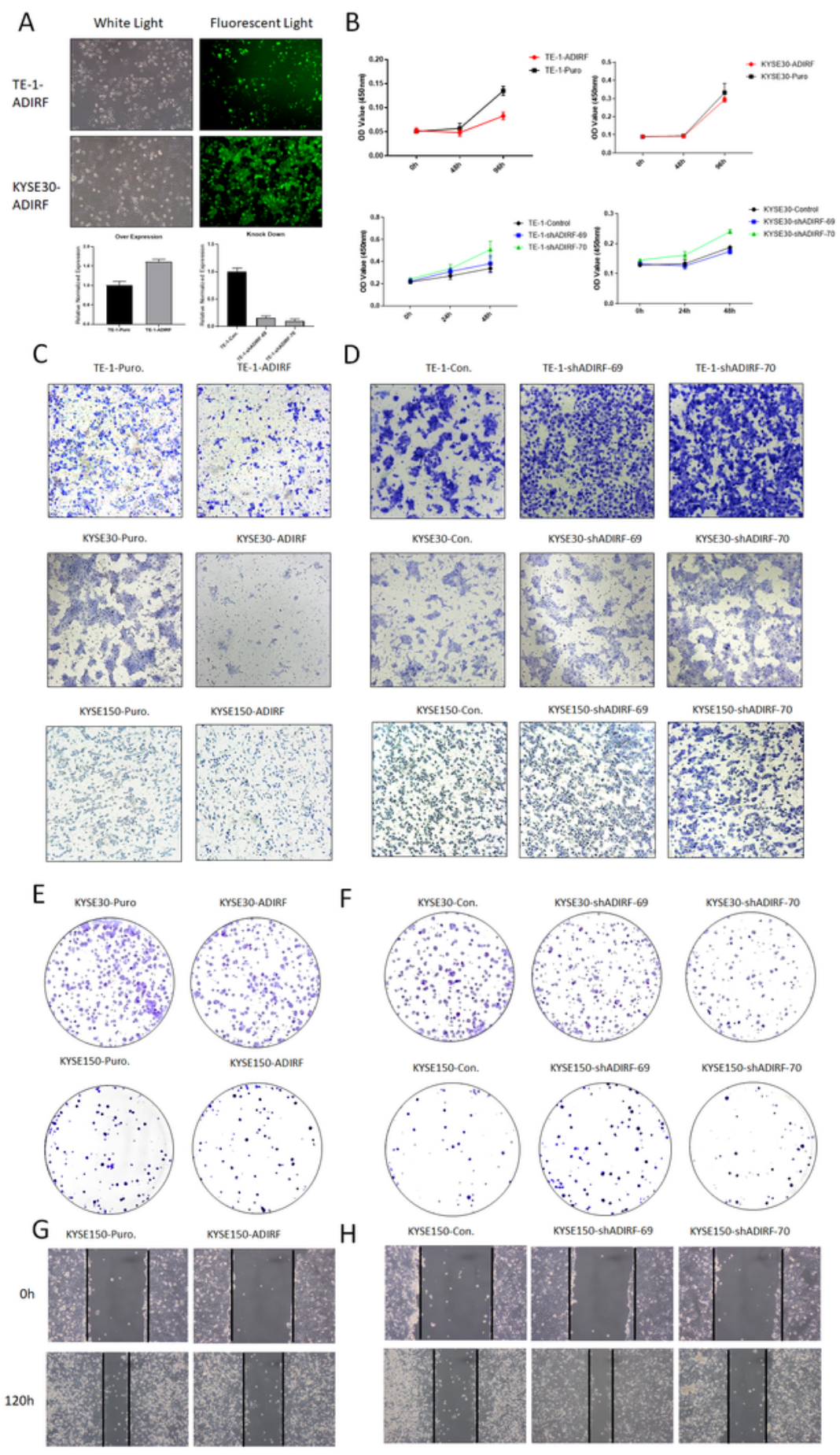

\section{Figure 3}

ADIRF inhibited tumor growth and migration in ESCC cells. We established the ADIRF-overexpressed cell lines (Co-expression with GFP) and ADIRF-suppressed ESCC cell lines (not shown) by infected with retroviruses and screened with puromycin. The histogram represented the mRNA level changes in the ADIRF-overexpressed cell lines (left) and ADIRF-suppressed cell lines (right) according to Q-PCR (A). The overexpression/suppression of ADIRF inhibited/promoted the proliferations in ESCC cells (B). The ADIRF 
overexpression reduced the capability of invasion (C) and the suppression increased the capability of invasion in three paired ESCC cell lines (D). The ADIRF overexpression inhibited the clone forming abilities (E) and the suppression enhanced it except for KYSE30 (F). G, In KYSE150 cell line, ADIRF overexpression / suppression decreased / increased the capability of migration indicated by healing assay (G-H).

Figure 4

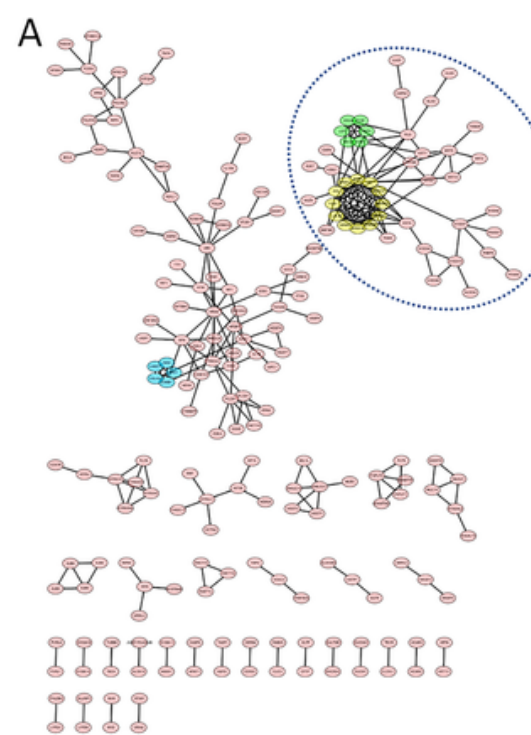

B

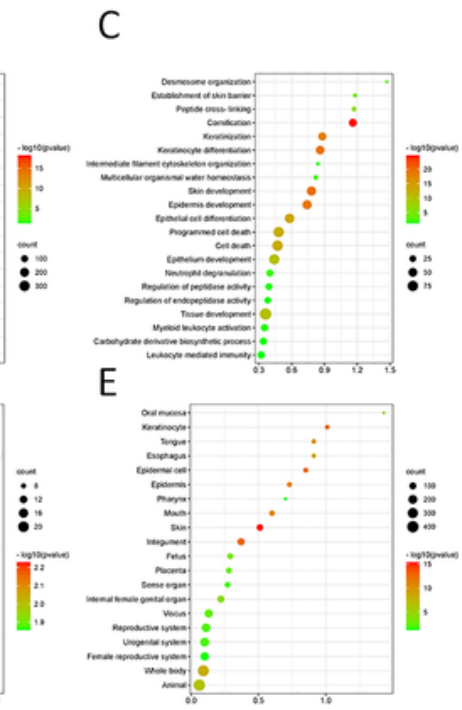

$\mathrm{F}$
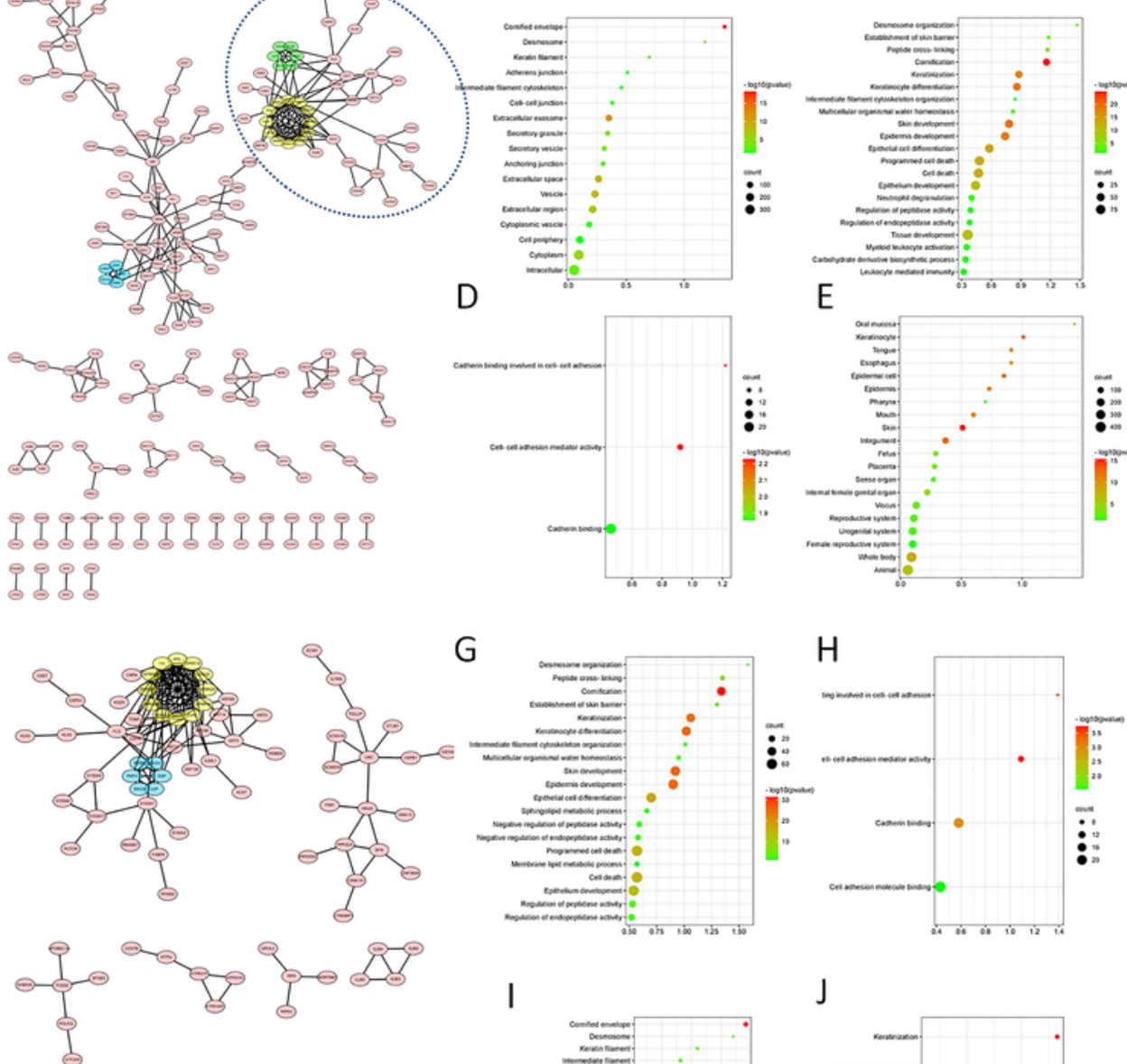

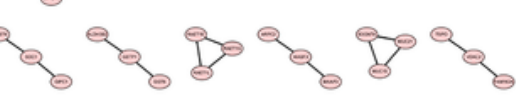

दू.

잉ํㅇำ

K
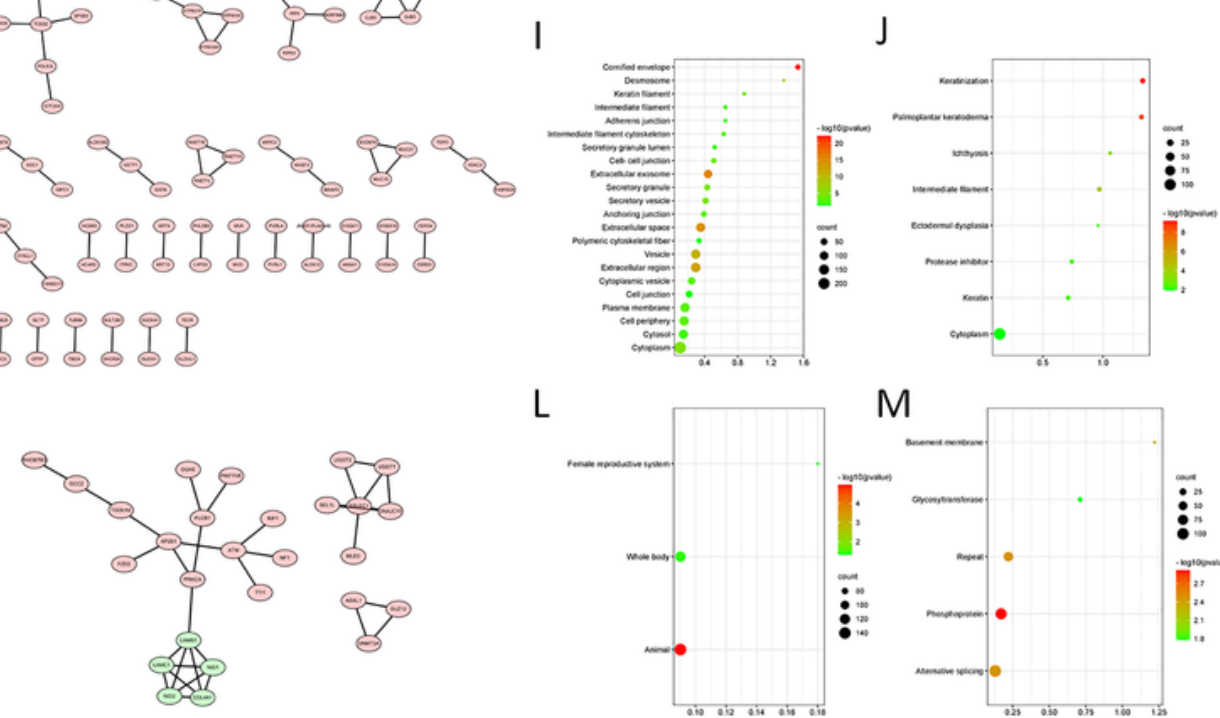

L
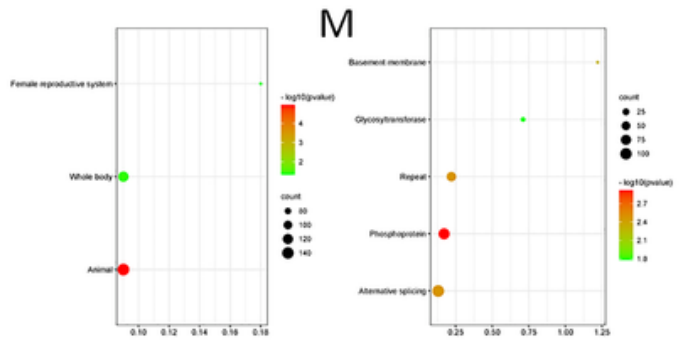

Figure 4 
ADIRF were associated with keratinocyte differentiation ESCC. A. interaction network of ADIRF correlated genes in ESCC. Functional analysis showed the most enriched functions of ADIRF correlated genes in the terms of cellular component (B), biological process (C), molecular functions (D) and the tissue specificity (E). F, interaction network of ADIRF positively correlated genes in ESCC. Functional analysis showed the most enriched functions of ADIRF positively correlated genes in the terms of cellular component $(G)$, biological process $(H)$, molecular functions $(I)$ and the pathway $(J)$. $K$, interaction network of ADIRF negative correlated genes in ESCC. Functional analysis indicated the most enriched functions of ADIRF negatively correlated genes in the terms of tissues specificity $(L)$ and the key words $(M)$.

\section{Figure 5}

\section{SPRR1A was a core regulator of keratinocyte differentiation and reversely correlated with ESCC}

progression. The overall 46 keratinocyte differentiation associated genes were unevenly expressed among different AJCC stages (A) and significantly downregulated in the advanced stages (B). The strong correlations were shown between keratinocyte differentiation associated genes and ADIRF (C). Interaction network indicated that the genes of SPRR1A and SPRR1B were located at the functional center (D). The mutual correlations between keratinocyte differentiation associated genes and AJCC stage (D). According to COX regression analysis, five genes of SPRR1A, SPRR1B, PKP3, SPRR2D, and FLG were significantly correlated with the prognosis in ESCC (F). The expression of SPRR1A was observably correlated with ADIRF $(G)$ and significantly downregulated in the advanced ESCCs $(H) .\left({ }^{*}: P<0.05 ;{ }^{* *}\right.$ : $\left.\mathrm{P}<0.01 ;{ }^{* \star *}: \mathrm{P}<0.001 ;{ }^{* * *}: \mathrm{P}<0.0001\right)$

\section{Supplementary Files}

This is a list of supplementary files associated with this preprint. Click to download.

- SupplementaryTables.docx 